\title{
Langmuir
}

pubs.acs.org/Langmuir

(C) 2010 American Chemical Society

\section{Time-Resolved Ultrathin Cobalt Film Growth on a Colloidal Polymer Template}

\author{
Adeline Buffet,* Mottakin M. Abul Kashem, Kai Schlage, Sébastien Couet, Ralph Röhlsberger, \\ André Rothkirch, Gerd Herzog, Ezzeldin Metwalli, Robert Meier, Gunar Kaune, Monica Rawolle, \\ Peter Müller-Buschbaum, Rainer Gehrke, and Stephan V. Roth

\begin{abstract}
HASYLAB-DESY, Notkestr. 85, 22607 Hamburg, Germany, K. U. Leuven Afdeling Kern en Stralingsfysica, 3001 Heverlee, Belgium, and, TU München, Physik-Department LS E13, James-Franck-Str. 1,
\end{abstract} \\ 85747 Garching, Germany
}

Received July 21, 2010. Revised Manuscript Received September 13, 2010

\begin{abstract}
Cobalt (Co) sputter deposition onto a colloidal polymer template is investigated using grazing incidence small-angle X-ray scattering (GISAXS), scanning electron microscopy (SEM), and atomic force microscopy (AFM). SEM and AFM data picture the sample topography, GISAXS the surface and near-surface film structure. A two-phase model is proposed to describe the time evolution of the Co growth. The presence of the colloidal template results in the correlated deposition of an ultrathin Co film on the sample surface and thus in the creation of Co capped polystyrene (PS) colloids. Well below the percolation threshold, the radial growth is restricted and only height growth is observed.
\end{abstract}

\section{Introduction}

Polymer-metal nanocomposite materials are outstanding materials for basic research and industrial applications. Recently, new applications for metallized polymer films and membranes have emerged in the field of organic electronics with the realization of organic transistors ${ }^{1,2}$ and organic solar cells, ${ }^{3-5}$ or in the field of hybrid sensors with the development of functional polymer-metal nanocomposite films ${ }^{6}$ and the development of membranes for high flux gas transport. ${ }^{7}$ Therefore, much effort has been put into better understanding of the growth process and structural transition of nanocomposites. ${ }^{8,9}$ It has been shown that the optical and electrical properties of a metal-polymer film are very sensitive to small changes in the composition and/or morphology of the film and that this sensitivity is drastically increased near the percolation threshold, ${ }^{10}$ when a conducting path is formed for the first time.

*To whom correspondence should be addressed. E-mail: adeline.buffet@ desy.de.

(1) Muccini, M. Nat. Mater. 2006, 5, 605-613.

(2) Dionigi, C.; Stoliar, P.; Porzio, W.; Destri, S.; Cavallini, M.; Bilotti, I.; Brillante, A.; Biscarini, F. Langmuir 2007, 23, 2030-2036.

(3) Perlich, J.; Memesa, M.; Diethert, A.; Metwalli, E.; Wang, W.; Roth, S. V.; Timmann, A.; Gutmann, J. S.; Müller-Buschbaum, P. ChemPhysChem 2009, 10, $799-805$.

(4) Lechmann, M. C.; Kessler, D.; Gutmann, J. S. Langmuir 2009, 25, 1020210208 .

(5) Tahk, D.; Kim, T.-I.; Yoon, H.; Moonkee, C.; Shin, K.; Suh, K. Y. Langmuir 2010, 26, 2240-2243

(6) Wolkenhauer, M.; Bumbu, G.-G.; Cheng, Y.; Roth, S. V.; Gutmann, J. S. Appl. Phys. Lett. 2006, 79, 054101.

(7) Kim, S.; Jinschek, J. R.; Chen, H.; Scholl, D. S.; Marand, E. Nano Lett. 2007, 7, 2807-2811.

(8) Roth, S. V.; Rothkirch, A.; Autenrieth, T.; Gehrke, R.; Wroblewski, T.; Burghammer, M. C.; Riekel, C.; Schulz, L.; Hengstler, R.; Müeller-Buschbaum, P. Langmuir 2009.

(9) Kaune, G.; Ruderer, M. A.; Metwalli, E.; Wang, W.; Couet, S.; Schlage, K.; Röhlsberger, R.; Roth, S. V.; Müller-Buschbaum, P. ACS Appl. Mater. Interfaces 2009, 1, 353-360.

(10) Schürmann, U.; Takele, H.; Zaporojtchenko, V.; Faupel, F. Thin Solid Films 2006, 515, 801-804.

(11) Roth, S. V.; Walter, H.; Burghammer, M.; Riekel, C.: Lengeler, B.; Schroer, C.; Kuhlmann, M.; Walther, T.; Sehrbrock, A.; Domnick, R.; Müller-Buschbaum, P. Appl. Phys. Lett. 2006, $88,021910$.

(12) Metwalli, E.; Couet, S.; Schlage, K.; Röhlsberger, R.; Körstgens, V.; Ruderer, M. A.; Wang, W.; Kaune, G.; Roth, S. V.; Müller-Buschbaum, P. Langmuir 2008, 24, 4265-4272.
Different techniques are available for producing composite materials. To name just a few, there are vacuum deposition, ${ }^{9-12}$ molecular beam epitaxy, ${ }^{13}$ pulsed laser deposition, ${ }^{14,15}$ dip coating, ${ }^{16-18}$ and solution casting. ${ }^{19,20}$ In general, vacuum deposition of metallic material is the most widely used technique in industrial applications. $^{21}$

The aim of our study is to investigate in situ and in real time the growth of an ultrathin metallic layer on a colloidal polymer template. Colloidal templates have been shown to be promising candidates for the generation of ultra-high-density media for magnetic data storage ${ }^{22}$ and to offer new possibilities in the development of solar cells. ${ }^{23}$ Investigation of the film composition and morphology and understanding of its growth are mandatory for a better control of the deposition process and thus of the metallized polymer film final properties. Our approach focuses on a three-layer system, namely, silicon ( $\mathrm{Si}$ ) substrate/polystyrene (PS) colloids/cobalt (Co), which is scalable as desired in many applications. ${ }^{24} \mathrm{We}$ will show that the existence of the colloidal template results in the correlated growth of an ultrathin Co film, which exactly reproduces the polymer template topography.

(13) Okuda, H.; Ochiai, S.; Ito, K.; Amemiya, Y. Appl. Phys. Lett. 2002, 81, 2358-2360.

(14) Siegal, M. P.; Yelton, W. G.; Overmeyer, D. L.; Provencio, P. P. Langmuir 2004, 20, 1194-1198

(15) Röder, J.; Faupel, J.; Krebs, H.-U. Appl. Phys. A: Mater. Sci. Processing 2008, 93, 863-867.

(16) Ferrer, M.; Lianos, P. Langmuir 1996, 12, 5620-5624

(17) Xia, S. J.; Liu, G.; Birss, V. I. Langmuir 2000, 16, 1379-1387.

(18) Frömsdorf, A.; Capek, R.; Roth, S. V. J. Phys. Chem. B 2006, 110, 15166 15171 .

(19) Roth, S. V.; Autenrieth, T.; Gruebel, G.; Riekel, C.; Burghammer, M.; Hengstler, R.; Schulz, L.; Müller-Buschbaum, P. Appl. Phys. Lett. 2007, 91 , 091915 .

(20) Shah, S. A.; Nag, M.; Kalagara, T.; S., S.; Manorama, S. V. Chem. Mater. 2008, 20, 2455-2460.

(21) Biswas, B.; Karulkar, P.; Eilers, H.; Norton, M.; Skorski, D.; Davitt, C.; Greve, H.; Schürmann, U.; Zaporojtchenko, V.; Faupel, F. Vac. Technol. Coat. 2006, 54-59.

(22) Park, S.; Lee, D. H.; Xu, J.; Kim, B.; Hong, S.; Jeong, U.; Xu, T.; Russell, T. P. Science 2009, 323, 1030-1033.

(23) Kaune, G.; Memesa, M.; Ruderer, M.; Diethert, A.; Roth, S. V.; D’Acunzi, M.; Gutmann, J. S.; Müeller-Buschbaum, P. ACS Appl. Mater. Interfaces 2009, 1, 2862-2869.

(24) Xia, Y.; Gates, B.; Yin, Y.; Lu, Y. Adv. Mater. 2000, 12, 693-713. 


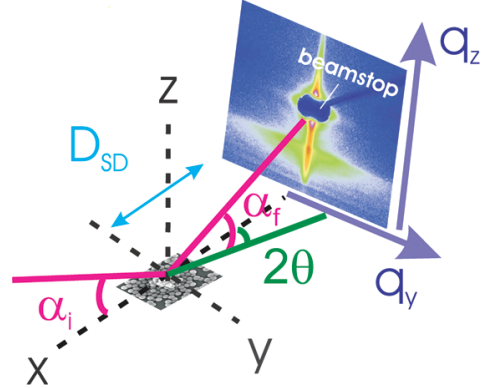

Figure 1. GISAXS setup geometry: $D_{\mathrm{SD}}$ denotes the sampleto-detector distance and $\alpha_{\mathrm{i}}$ and $\alpha_{\mathrm{f}}$ the incident and exit angles, respectively. A beamstop is used to protect the detector from the reflected beam.
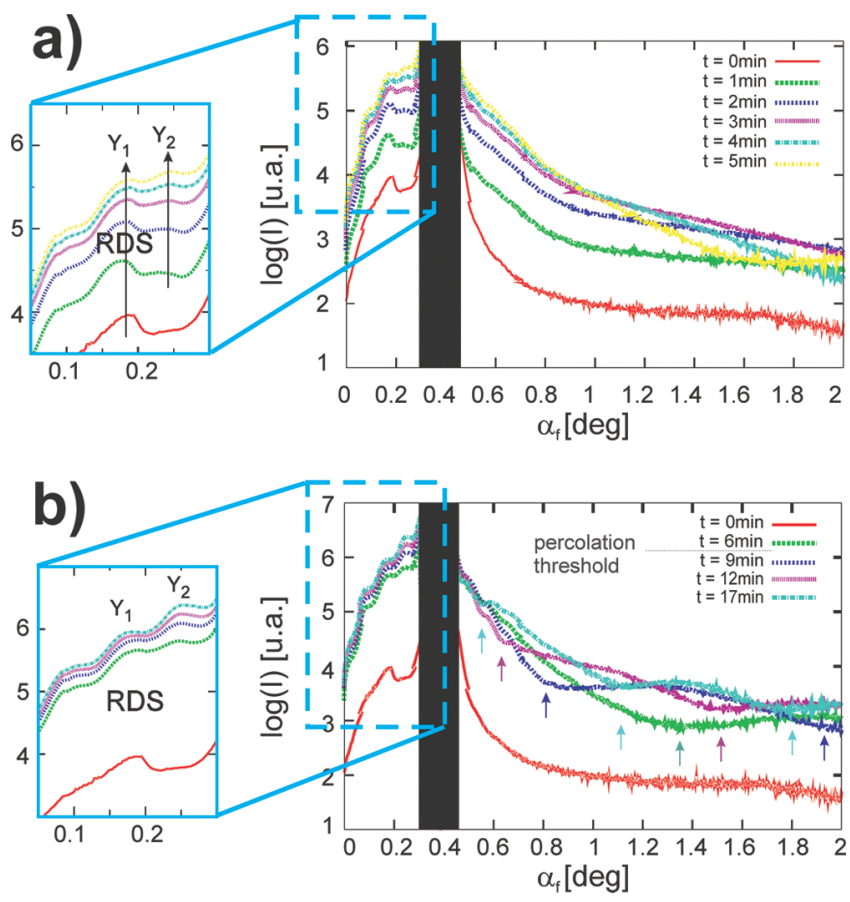

Figure 2. Evolution of the detector cut $\left(2 \theta=0^{\circ}\right)$ during Co sputter deposition sputtering. (a) $t=0,1,2,3,4$, and $5 \mathrm{~min}$. (b) $t=0,6,9,12$, and $17 \mathrm{~min}$. Yoneda peaks of $\operatorname{Si}\left(Y_{1}\right)$ and $\operatorname{Co}\left(Y_{2}\right)$ and intensity modulations due to resonant diffuse scattering (RDS) are indicated. The rectangular (black) area corresponds to the specular beamstop position as can be seen in Figure 1 .

\section{Results and Discussion}

The template was prepared by spin-coating a PS colloidal solution (G. Kisker GbR, concentration $0.025 \%$, nominal particle diameter of $100 \mathrm{~nm}$ ) on top of a precleaned Si substrate. No additional processing was applied.

Complementary techniques were used to investigate the growth of Co onto the colloidal polymer template: in situ microfocus beam grazing incidence small angle X-ray scattering $\left(\mu\right.$-GISAXS $\left.{ }^{25}\right)$, scanning electron microscopy (SEM), and atomic force microscopy (AFM). While AFM and SEM-being local probing techniques - allow for a local investigation of the surface structure, GISAXS allows for the examination of a larger area and thus provides statistically significant information to prove largescale structural homogeneity.

(25) Roth, S. V.; Döhrmann, R.; Dommach, M.; Kuhlmann, M.; Kröger, I.; Gehrke, R.; Walter, H.; Schroer, C.; Lengeler, B.; Müller-Buschbaum, P. Rev. Sci. Instrum. 2006, 77, 085106.
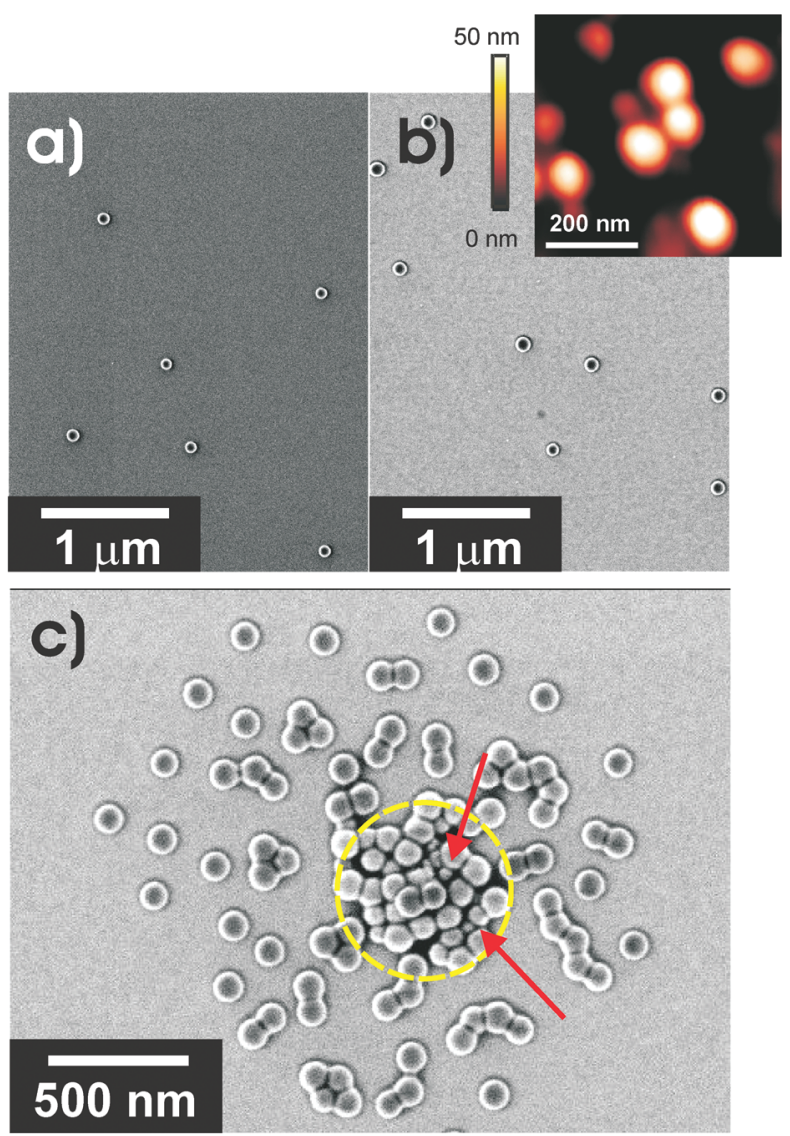

Figure 3. SEM images of the sample before (a) and after $(b, c)$ deposition of $12 \mathrm{~nm}$ (nominal) Co. Better contrast is observed in the images of the Co-coated sample. The circle in (c) denotes the agglomerated colloids. The arrows hightlight the presence of few smaller colloids. Inset: AFM topographic image of the coated sample.

In situ stop-sputter ${ }^{12}$ experiments were carried out at the beamline BW4 ${ }^{25}$ (HASYLAB, Germany) at a wavelength $\lambda=0.138 \mathrm{~nm}$. The stop-sputter procedure comprises sputter deposition and subsequent GISAXS measurement. A dedicated ultra-high-vacuum sputter deposition chamber was mounted at the beamline. ${ }^{26} \mathrm{Co}$ sputter depositions were performed with a deposition rate of $(5 \pm 1) \AA / \mathrm{min}$ at an argon pressure of $P=4 \times 10^{-3} \mathrm{mbar}$. A schematic drawing of the GISAXS geometry is shown in Figure 1. We used an angle of incidence $\alpha_{i}=0.4^{\circ}$ and a sample-to-detector distance $D_{\mathrm{SD}}=2.21 \mathrm{~m}$.

Structural information is obtained from vertical $I\left(q_{z}\right)$ and horizontal $I\left(q_{y}\right)$ cuts of the 2D-intensity distribution, with $q_{z}$ and $q_{y}$

$$
q_{z}=\frac{2 \pi}{\lambda}\left(\sin \alpha_{\mathrm{i}}+\sin \alpha_{\mathrm{f}}\right) \quad \text { and } \quad q_{y}=\frac{2 \pi}{\lambda} \sin 2 \theta
$$

where $\alpha_{\mathrm{f}}$ denotes the exit angle in the $\left(\mathbf{q}_{x}, \mathbf{q}_{z}\right)$-plane and $2 \theta$ the exit angle in the $\left(\mathbf{q}_{x}, \mathbf{q}_{y}\right)$-plane, with $q_{x}=(2 \pi / \lambda)\left(\cos 2 \theta \cos \alpha_{\mathrm{f}}-\cos \alpha_{\mathrm{i}}\right)$, $\mathbf{q}_{x}$ being parallel to the X-ray beam axis.

Figure 2 shows the detector cuts extracted from the GISAXS patterns recorded before $(t=0 \mathrm{~min})$ and during Co sputter deposition ( $t=1,2,3,4,5,6,9,12$, and $17 \mathrm{~min})$. A strong increase in the scattered intensity is observed with Co sputter deposition. $Y_{1}$ and $Y_{2}$ indicate the Yoneda maxima of $\mathrm{Si}$ and Co, respectively. We note the following features. During the first minutes of $\mathrm{Co}$

(26) Couet, S.; Diederich, T.; Schlage, K.; Röhlsberger, R. Rev. Sci. Instrum. 2008, 79, 093908 . 

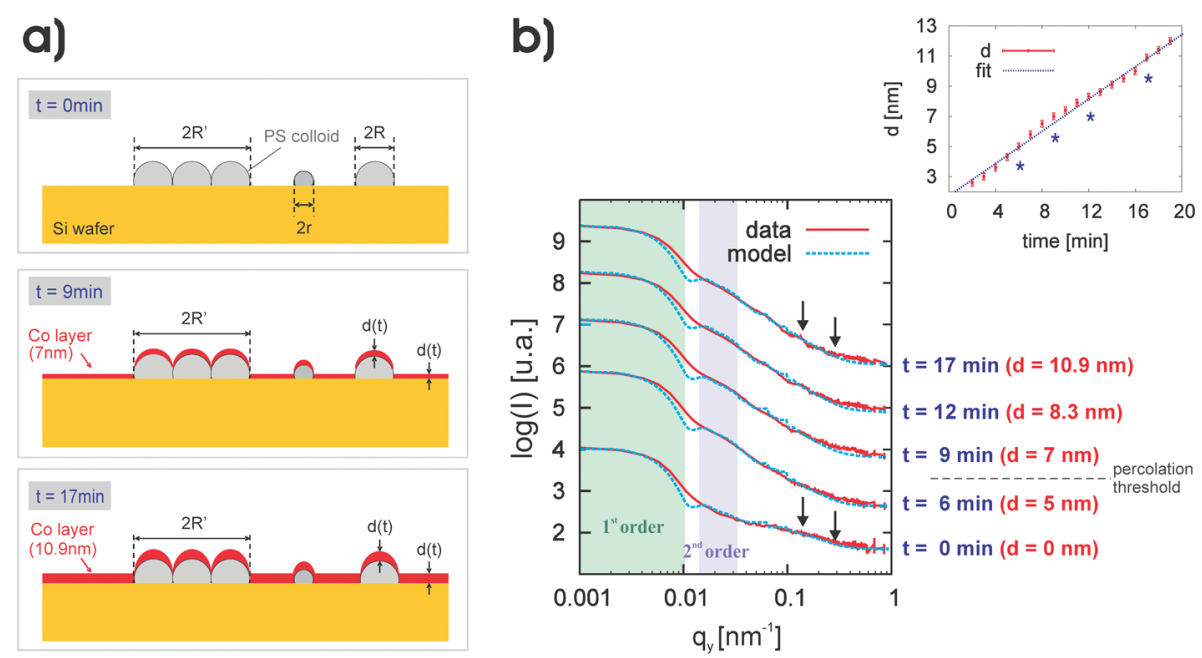

Figure 4. (a) Sketch of the system before $(t=0 \mathrm{~min})$ and during $(t>0 \mathrm{~min})$ Co sputter deposition. PS colloids are described as nanospheres $(R, r)$ that can form agglomerates (typical lateral dimension $\left.2 R^{\prime}\right)$. For $t>0$ min, we assumed the growth of a Co layer with thickness $d(t)$. (b) Experimental (solid red lines) and simulated (short dashed blue lines) out-of-plane cuts $\left(\alpha_{\mathrm{f}}=0.288^{\circ}\right)$ for $t=0,6,9,12$, and 17 min. The cuts were shifted for better visibility. The filled area outlines the first-order (green) and second-order (purple) maxima of the agglomerate form factor. Upper right: Time-resolved analysis of the Co layer thickness $d(t)$. The linear approximation indicates a growth rate of $(5 \pm 1) \AA /$ min. Times corresponding to the out-of-plane cuts are marked with by an asterisk.

sputter deposition ( $1 \leq t \leq 5 \mathrm{~min}$ ), we note a strong increase in $Y_{2}$ and in the resonant diffuse scattering (RDS) (see Figure $2 \mathrm{a}$ and enlargement). The strong interference peaks of the RDS visible in the region $0^{\circ} \leq \alpha_{\mathrm{f}} \leq 0.3^{\circ}$ are the signature of a strong correlation between the ultrathin growing Co film and the underlying colloidal polymeric template. Starting from $t \geq 5 \mathrm{~min}$, we observe the emergence of strong minima in the region $\alpha_{f} \geq 0.4^{\circ}$. Those minima shift to smaller $\alpha_{\mathrm{f}}$-values with increasing amount of deposited Co (see the vertical arrows in Figure 2b). This result indicates the growth of a correlated ultrathin Co layer perpendicular to the sample surface.

The sample was also investigated by using SEM (Zeiss Gemini NVision 40, TU-Muenchen) and AFM (NTEGRA probe NanoLaboratory, HASYLAB Hamburg) before and after sputter deposition of $12 \mathrm{~nm}$ (nominal) of Cobalt (Figure 3).

SEM measurements were performed at different sample positions using a voltage of $1 \mathrm{kV}$. The SEM data (Figure 3) highlight the presence of isolated PS colloids and agglomerated PS colloids. Typical lateral dimension of the colloids and agglomerates, as determined by SEM, are $(100 \pm 20) \mathrm{nm}$ and $(500 \pm 200) \mathrm{nm}$, respectively. Few smaller colloids with typical diameter of $(20 \pm 8)$ $\mathrm{nm}$ are also observed. The SEM images of the surface after deposition of $12 \mathrm{~nm}$ (nominal) Co (Figure 3b,c) show better contrast and enhanced conductivity (no charging effect observed during SEM measurements) compared to the SEM images of the PS colloidal template before Co deposition, $t=0 \mathrm{~min}$ (Figure $3 \mathrm{a}$ ). These features are typical of ultrathin metallic layers and confirm the growth of an ultrathin Co film as already determined by the GISAXS data analysis.

AFM measurements were performed at different sample positions in semicontact mode. The inset in Figure 3 shows the topography of the sample surface after deposition of $12 \mathrm{~nm}$ (nominal) of Cobalt. The AFM data confirm the presence of colloids on the sample surface with typical diameter of $(100 \pm 20) \mathrm{nm}$.

To shed light on the Co growth kinetics, a two-phase model was established (Figure 4a). In this model, the system in its reference state $(t=0 \mathrm{~min})$ is described as an assembly of isolated and agglomerated PS colloids. The PS colloids are found to have a radius of $R=(60 \pm 10) \mathrm{nm}$. Additional intensity observed in the region $q_{y}=0.3 \mathrm{~nm}^{-1}$ was interpreted as stemming from smaller colloids with radius $r=(10 \pm 4) \mathrm{nm}$. As observed in SEM (Figure 3c), PS colloids can form agglomerates. Best agreement was found when considering agglomerates with lateral dimension $2 R^{\prime}=(600 \pm 200) \mathrm{nm}$. Compared to the SEM results, the slight difference might be the illustration of a larger size polydispersity. To describe the system during sputter deposition ( $t>0 \mathrm{~min})$, we assumed the growth of an ultrathin Co layer on both the PS colloids and the bare Si substrate. We thus considered a layer thickness $d(t)$ increasing with sputter deposition time $t$. The initial radius ( $R$ or $r$ ) of the PS colloids is preserved throughout $\mathrm{Co}$ sputter deposition as can be seen in (Figure 4a).

On the basis of this two-phase model and using the software IsGISAXS ${ }^{27}$ we computed $I\left(q_{y}, t\right)$ for each time $t$ during sputter deposition (Figure 4b). A very good agreement between the experimental and simulated curves can be observed before and during Co deposition, corroborating the model proposed above. The scattering intensity at low $q_{y}$-values $\left(q_{y} \leq\right.$ $0.02 \mathrm{~nm}^{-1}$ ) stems from large structures-in our case, the PS agglomerates. Following the numerical analysis, the two first maxima of the intensity around $q_{y}=0.001 \mathrm{~nm}^{-1}$ and $q_{y}=$ $0.02 \mathrm{~nm}^{-1}$ (filled areas in Figure 4b) are attributed to the form factor of the PS agglomerates.

Concerning the Co layer thickness $d(t)$, the time-resolved analysis shows a linear trend with a growth rate of $(5 \pm 1) \AA / \mathrm{min}$, which is in agreement with the sputter rate applied. It is worth noting that the lateral dimension of the PS colloids is retained throughout the Co film growth (see vertical arrows in Figure 4b), the signature of the highly correlated ultrathin film growth on top of the colloidal template.

Our work shows that it is possible to follow the growth of an ultrathin Co layer onto a colloidal template. Estimating the typical percolation threshold of Cobalt as similar to the one of Gold $^{9}$ and Silver ${ }^{10,28}$ at $(7 \pm 1) \mathrm{nm}$, we deduce the following: From a nominal deposited thickness of $(3 \pm 1) \mathrm{nm}$ on, the RDS is fully established (Figure 2a). This means that the stable correlated

(27) Lazzari, R. J. Appl. Crystallogr. 2002, 35, 406-421.

(28) Walter, H.; Bauer, G.; Domnick, R.; Jakopic, G.; Leitner, A. Opt. Eng. 2006, 45,103801 . 
ultrathin Co film growth starts well below the percolation threshold, and that only the height of the Co film increases.

In summary, sputter deposition of Co on PS colloid-coated $\mathrm{Si}$ substrate was investigated using the combination of SEM, AFM, and GISAXS techniques. GISAXS data reveal the growth of a correlated ultrathin Co film on both the bare Si subtrate and the PS colloids. Time-resolved analysis of the GISAXS data highlights the strong correlation between the growing Co layer and the underlying polymer template, which means a perfect reproduction of the colloidal template lateral structure and a wetting of Co on the PS colloid curved surface. Potential lateral growth of the colloids due to wetting of the Co could not be resolved by $\mu$-GISAXS. By performing in situ GISAXS measurement during sputter deposition, we show that the correlated ultrathin film growth starts well below the percolation threshold.

Acknowledgment. Portions of this research were carried out at the light source DORIS III at HASYLAB/DESY. DESY is a member of the Helmholtz Association (HGF). The authors thank A. Timmann and T. Schubert for their support at the beamline.

Supporting Information Available: Description of the microfocus beam GISAXS setup geometry. Parameters used for the simulation of the scattered intensity (IsGISAXS). This material is available free of charge via the Internet at http://pubs.acs.org. 